Supporting Information

\title{
Top-down Fabrication of Spatially Controlled Mineral Gradient \\ Scaffolds for Interfacial Tissue Engineering
}

Alexander J. Boys, Hao Zhou, Jordan B. Harrod, Mary Clare McCorry, Lara A. Estroff*,

Lawrence J. Bonassar*

*Corresponding Authors 
Supplemental Methods
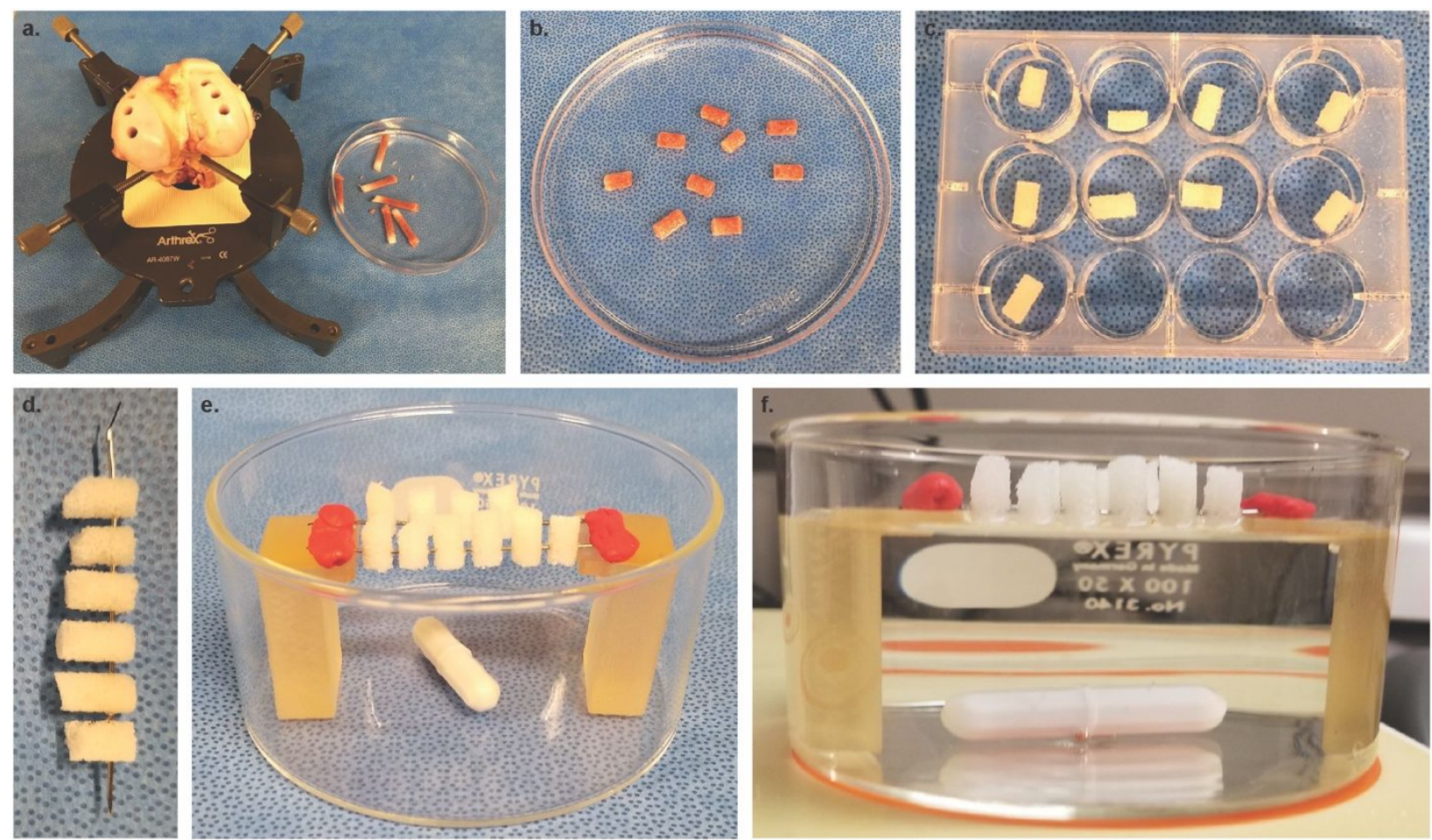

Figure S1. Demineralization procedure: a. explantation from bovine femur, b. explanted biopsies, c. decellularized biopsies, d. skewered biopsies, e. biopsies in demineralization setup, f. fluid level for demineralization front halfway along scaffold axis. 


\section{Determination of Length of Scaffolds}

Each sample subjected to a $\mu \mathrm{CT}$ scan was angled within the sample holder. To determine the length of each scaffold, the first and last z-slice containing an 'full' elliptical slice of the scaffold was found. A circle was drawn around these slices, determining the $\mathrm{x}, \mathrm{y}$, and $\mathrm{z}$ coordinates of the first and last 'full' z-slice and used to determine the length of the scaffold with the following equations:

$$
\begin{aligned}
& d=\sqrt{\left(x_{1}-x_{2}\right)^{2}+\left(y_{1}-y_{2}\right)^{2}+\left(z_{1}-z_{2}\right)^{2}} \\
& h=\sqrt{\left(z_{1}-z_{2}\right)^{2}} \\
& \theta=\arcsin (h / d) \\
& w=\frac{r}{\tan (\theta)} \\
& l=d+2 w
\end{aligned}
$$

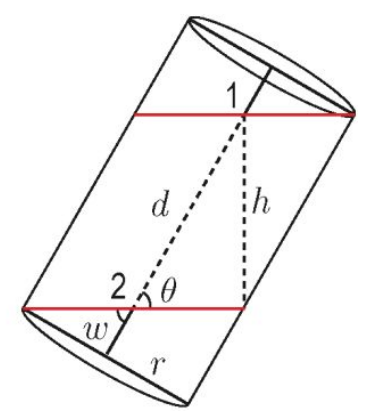

Figure S2. Equations and schematic to calculate length of scaffold oriented at an angle. 

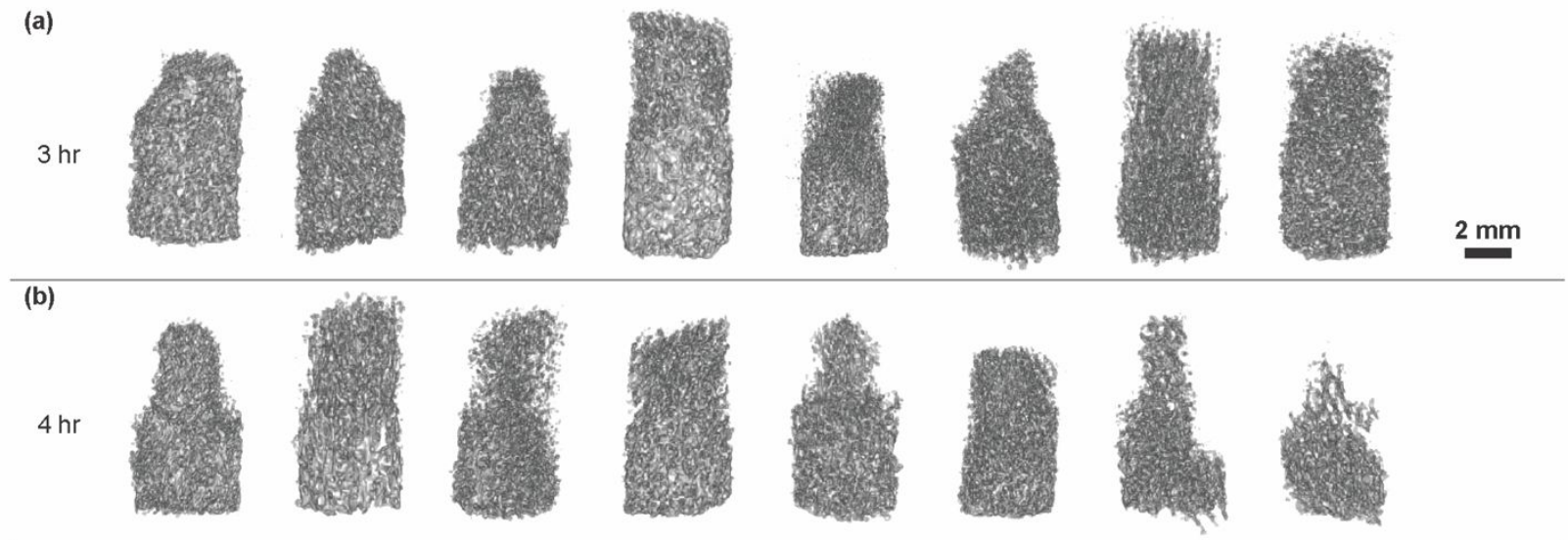

(c)
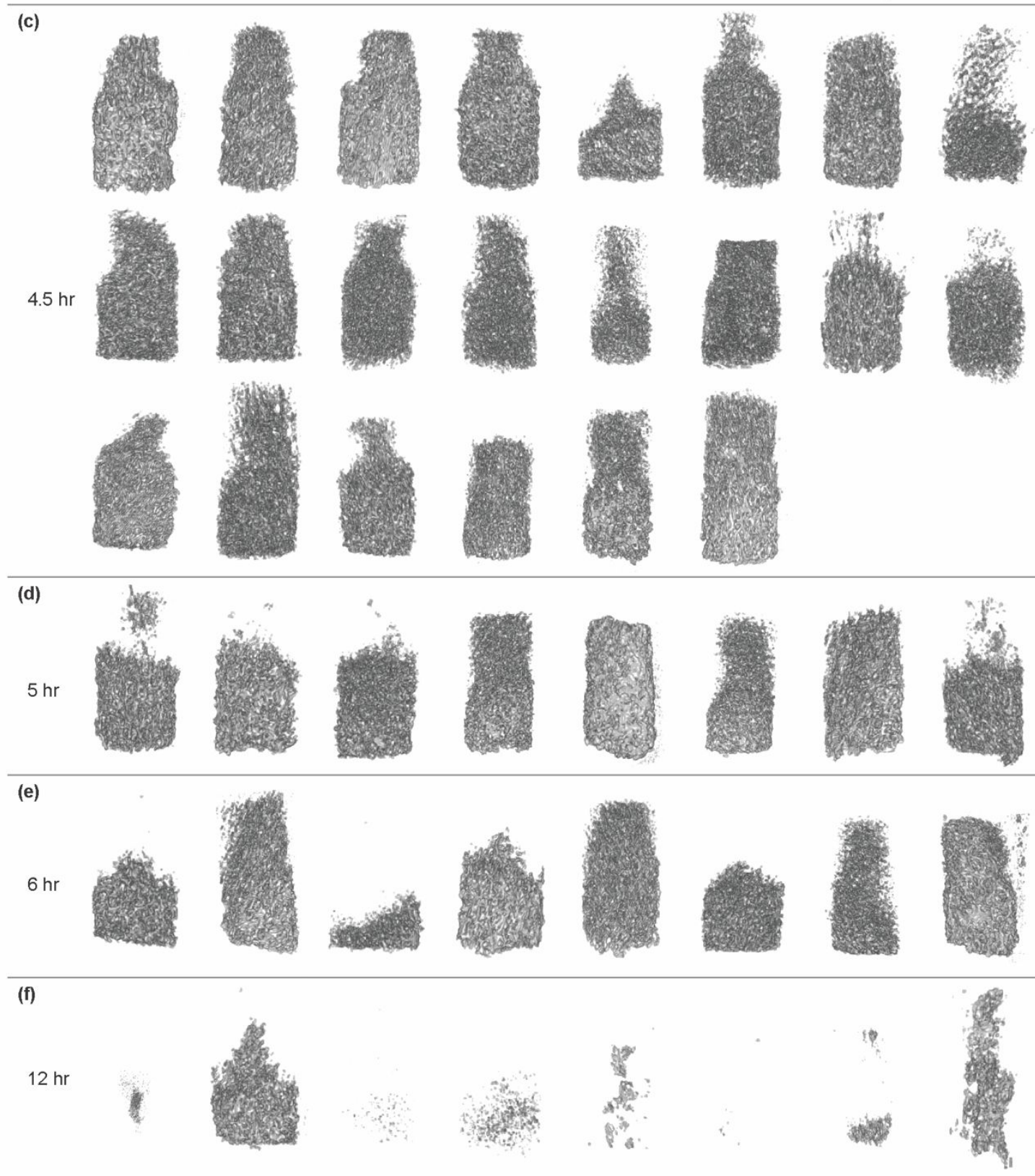
Figure S3. $\mu$ CT renderings of all fabricated interfacial scaffolds at (a) 3 hour time point, (b) 4 hour time point, (c) 4.5 hour time point, (d) 5 hour time point, (e) 6 hour time point, and (f) 12 hour time point.

(a)

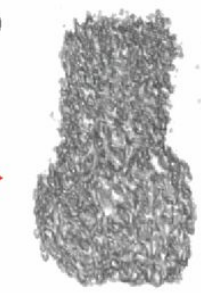

(b)

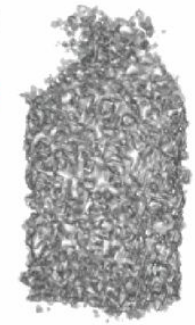

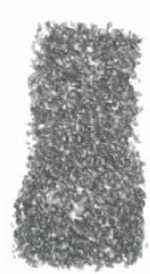
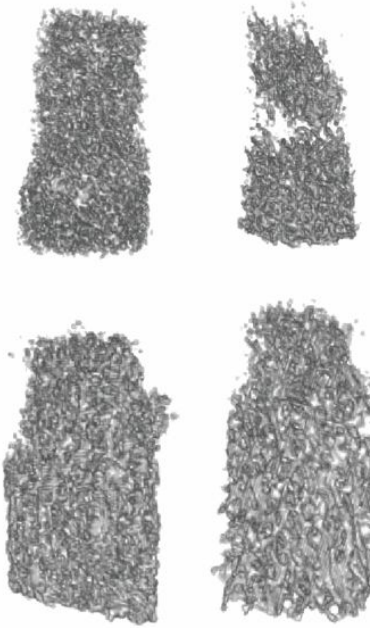
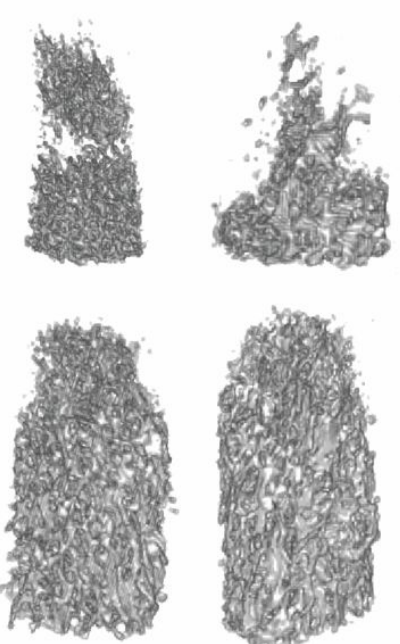
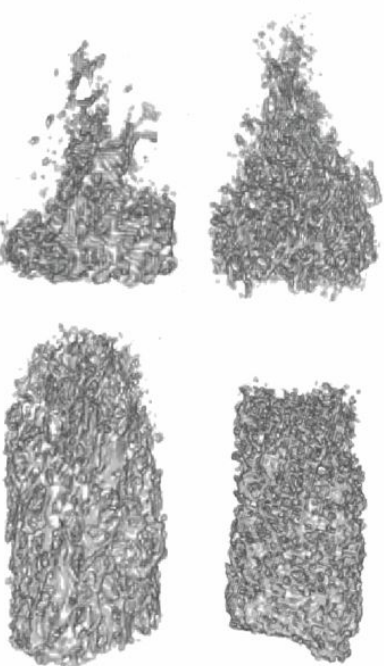
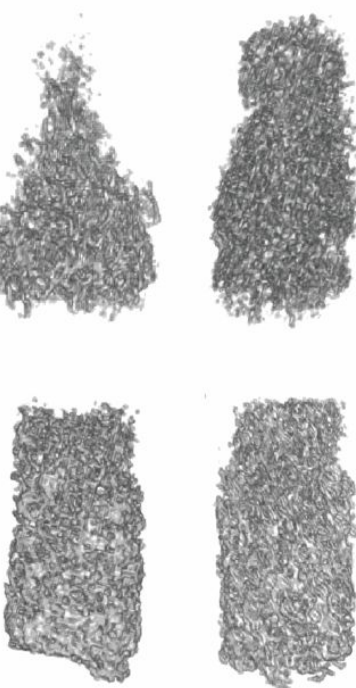

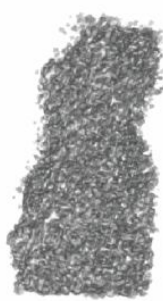

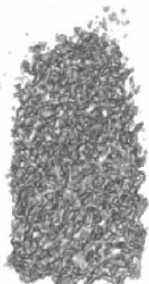

Figure S4. Rendered $\mu \mathrm{CT}$ images of interfacial scaffolds demineralized for 4.5 hours at different skewering locations. Red arrow indicates skewering location.

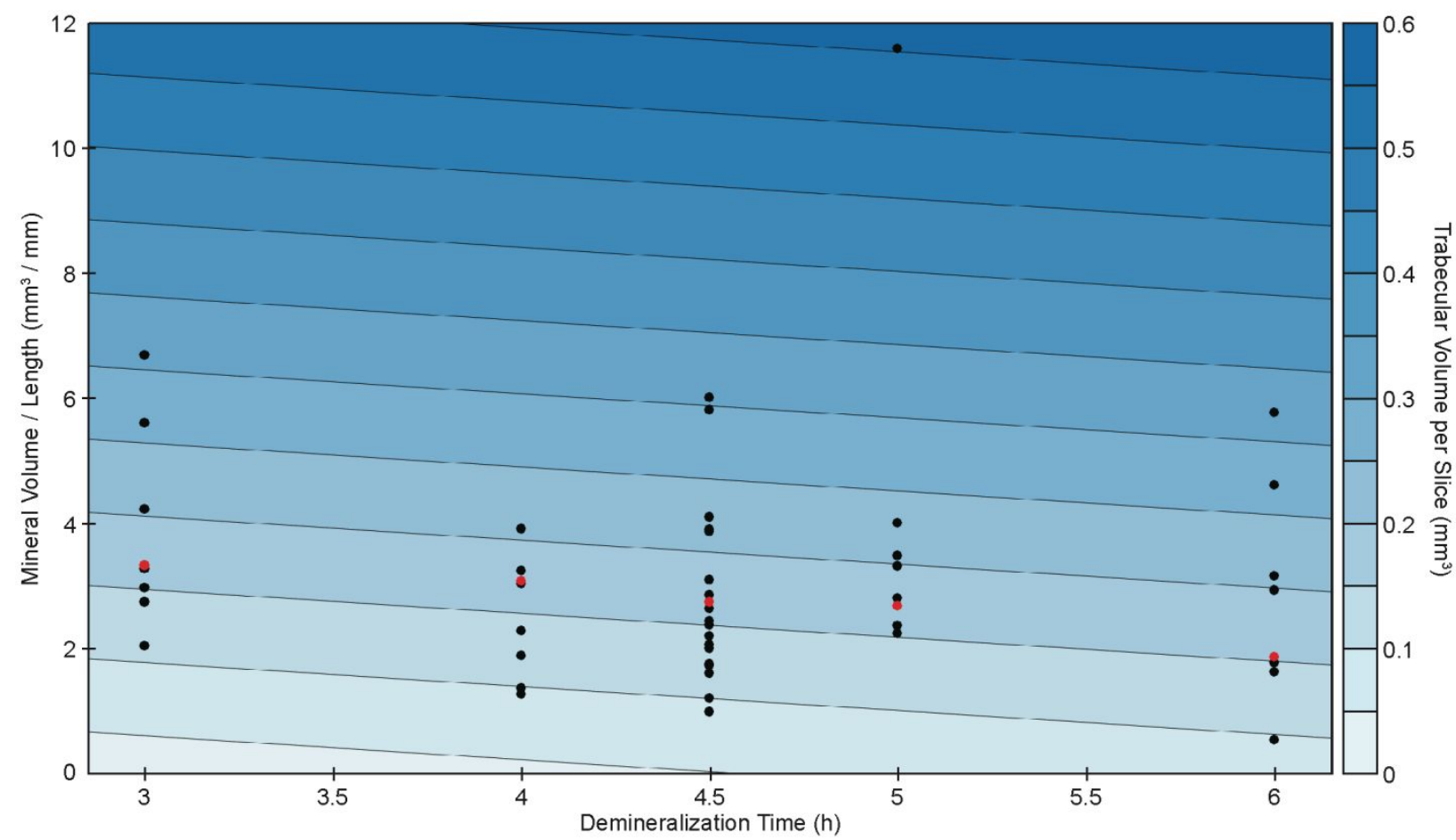

Figure S5. Alternate view of fitted surface for relationship of mineral volume / length to time to trabecular volume per slice from Figure $3 \mathrm{~b}$. Red dots show the chosen interfacial scaffolds in Figure 3a. 

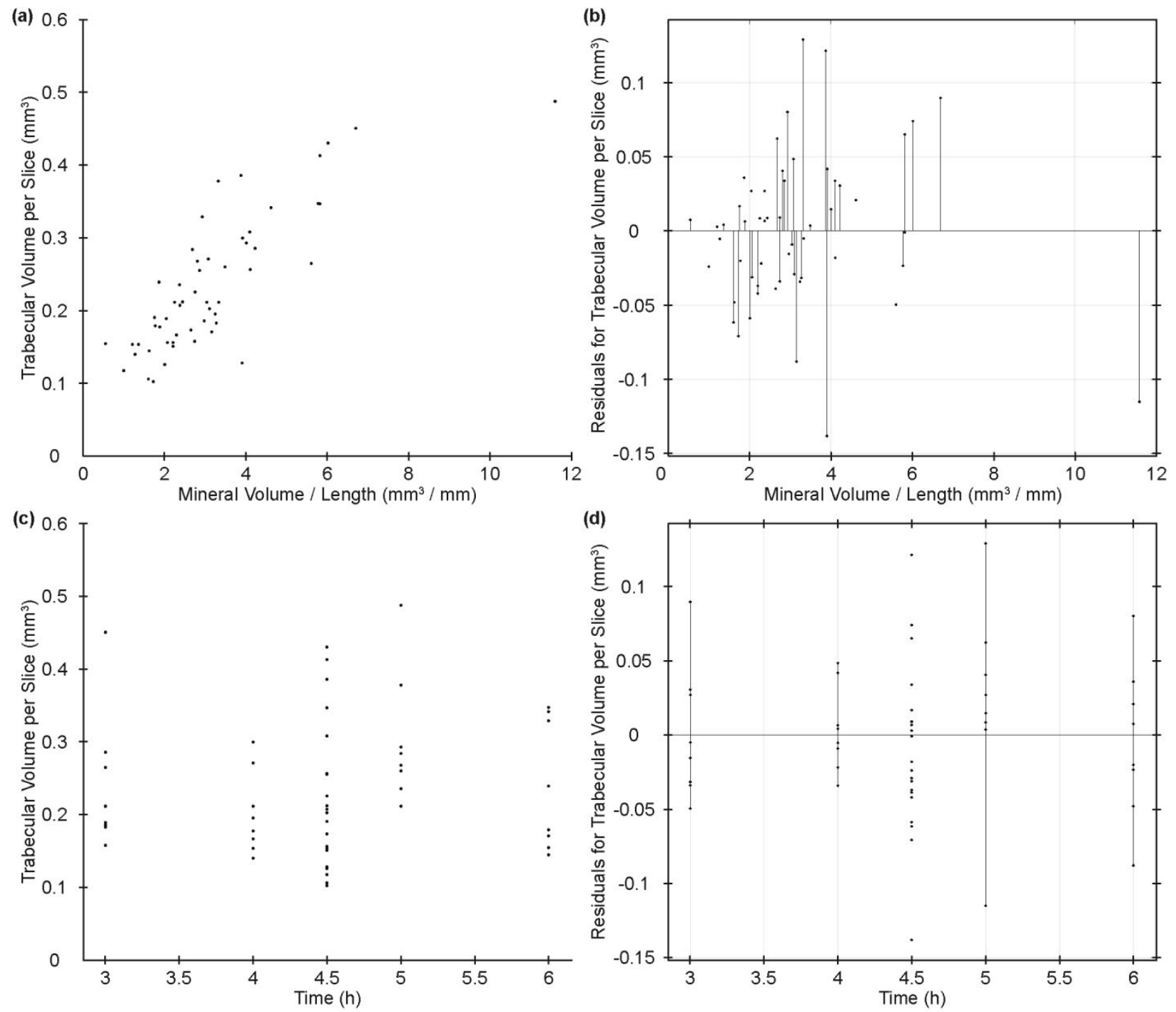

Figure S6. (a) Plot of trabecular volume per slice versus mineral volume / length with labeled axes. (b) Plot showing residuals for surface fit for mineral volume / length. (c) Plot of trabecular volume per slice versus time with labeled axes. (d) Plot of residuals for surface fit for time. 

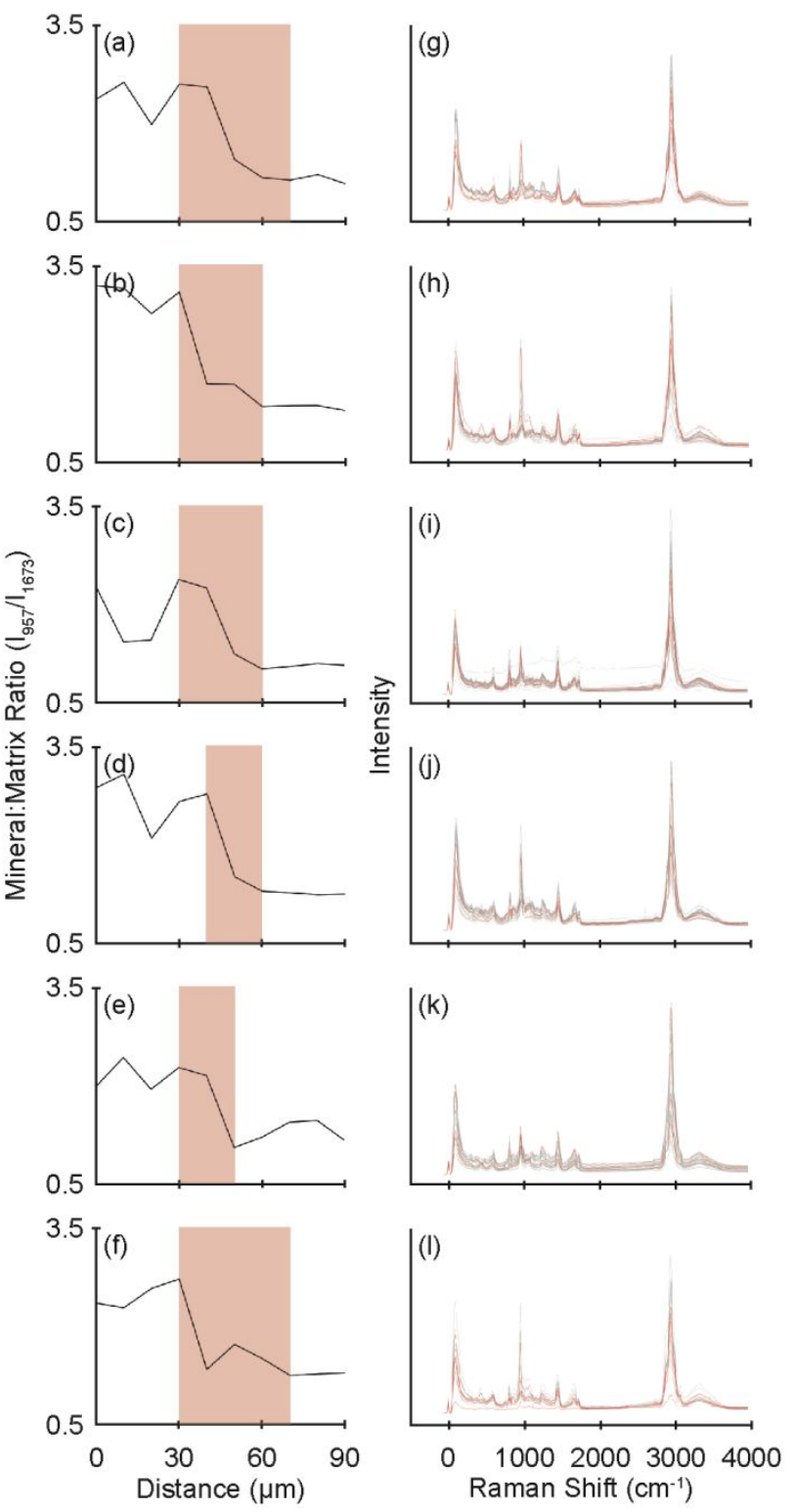

Figure S7. $(\mathrm{a}-\mathrm{f})$ Mineral:matrix gradient as a function of distance at the immediate interface between mineralized and demineralized tissue paired with $(\mathrm{g}-1)$ Raman spectra across line scan used to calculate mineral : matrix ratio $\left(957: 1673 \mathrm{~cm}^{-1}\right)$. Red shaded regions indicate gradient, and red spectra are within gradient region. Gray spectra are from region extending around gradient. 


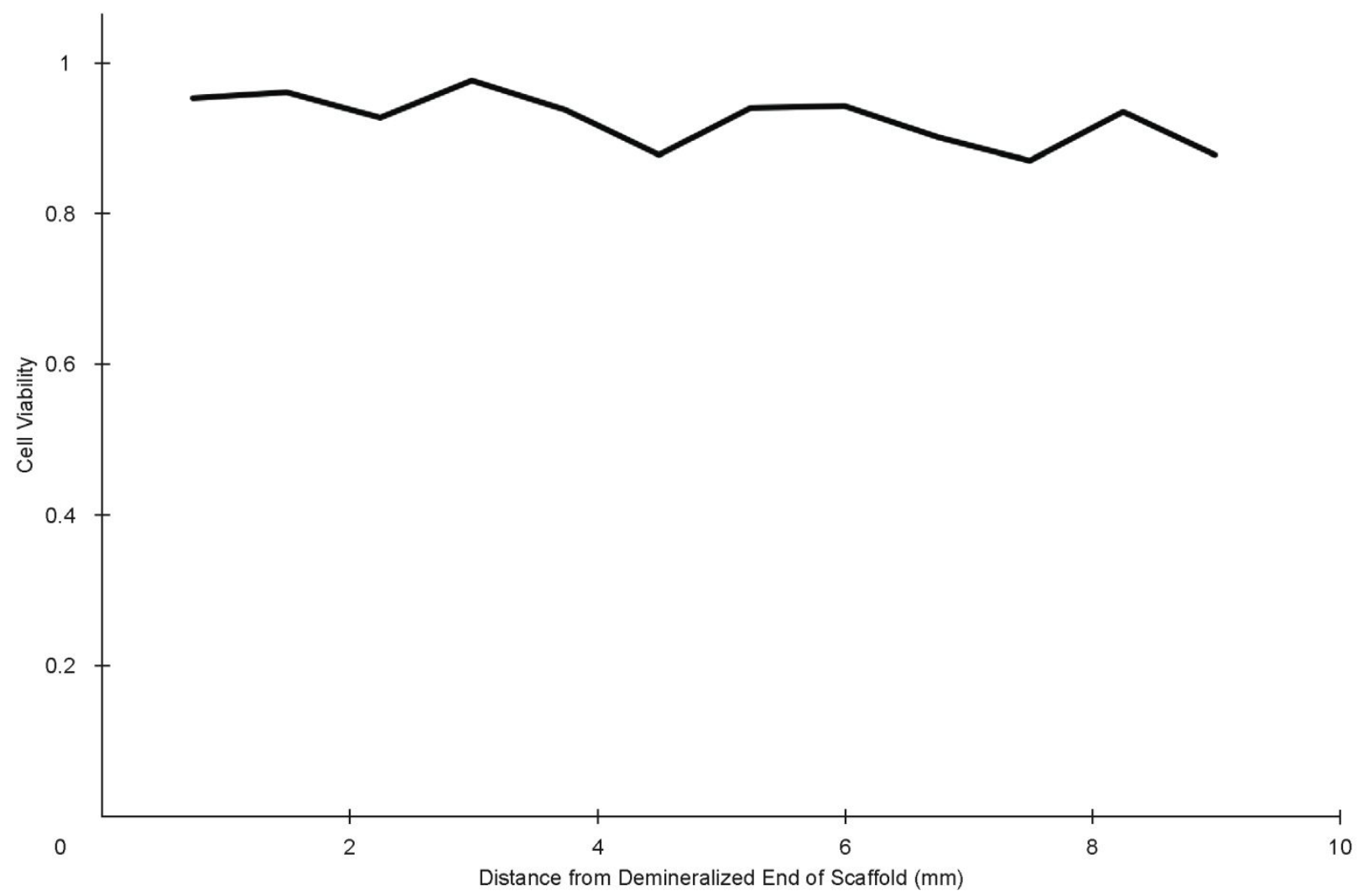

Figure S8. Representative plot of cell viability, from Live/Dead assay after seeding, as a function of distance across partially demineralized scaffold. The line represents the fraction of live cells versus total cells with data collected at $750 \mu \mathrm{m}$ increments from demineralized end of scaffold to mineralized end of scaffold. 


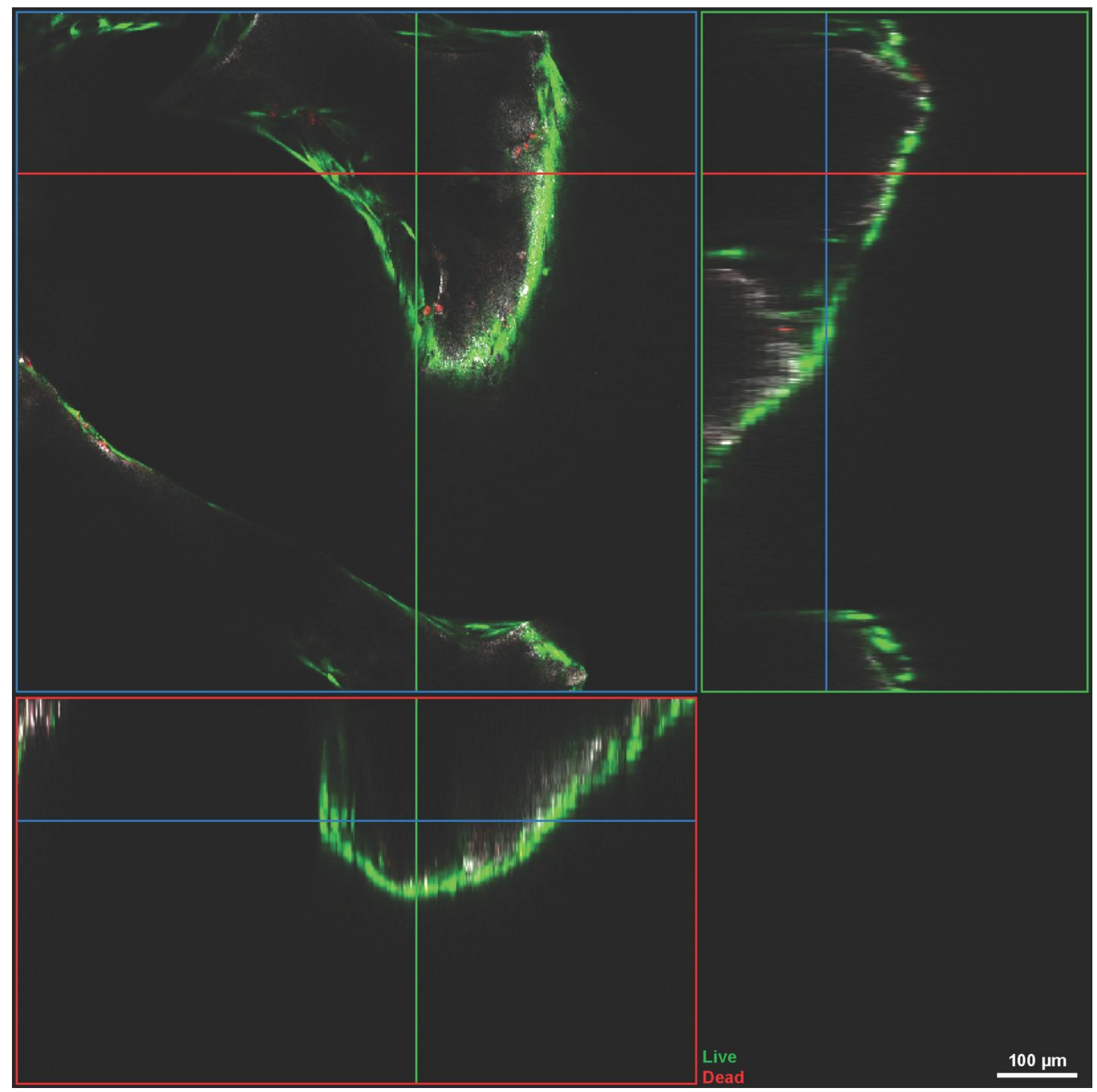

Figure S9. Confocal image of Live/Dead stain from z-stack in Figure 7a showing different slices of z-stack. Images show that cells are adhered to the exterior of the trabeculae. 

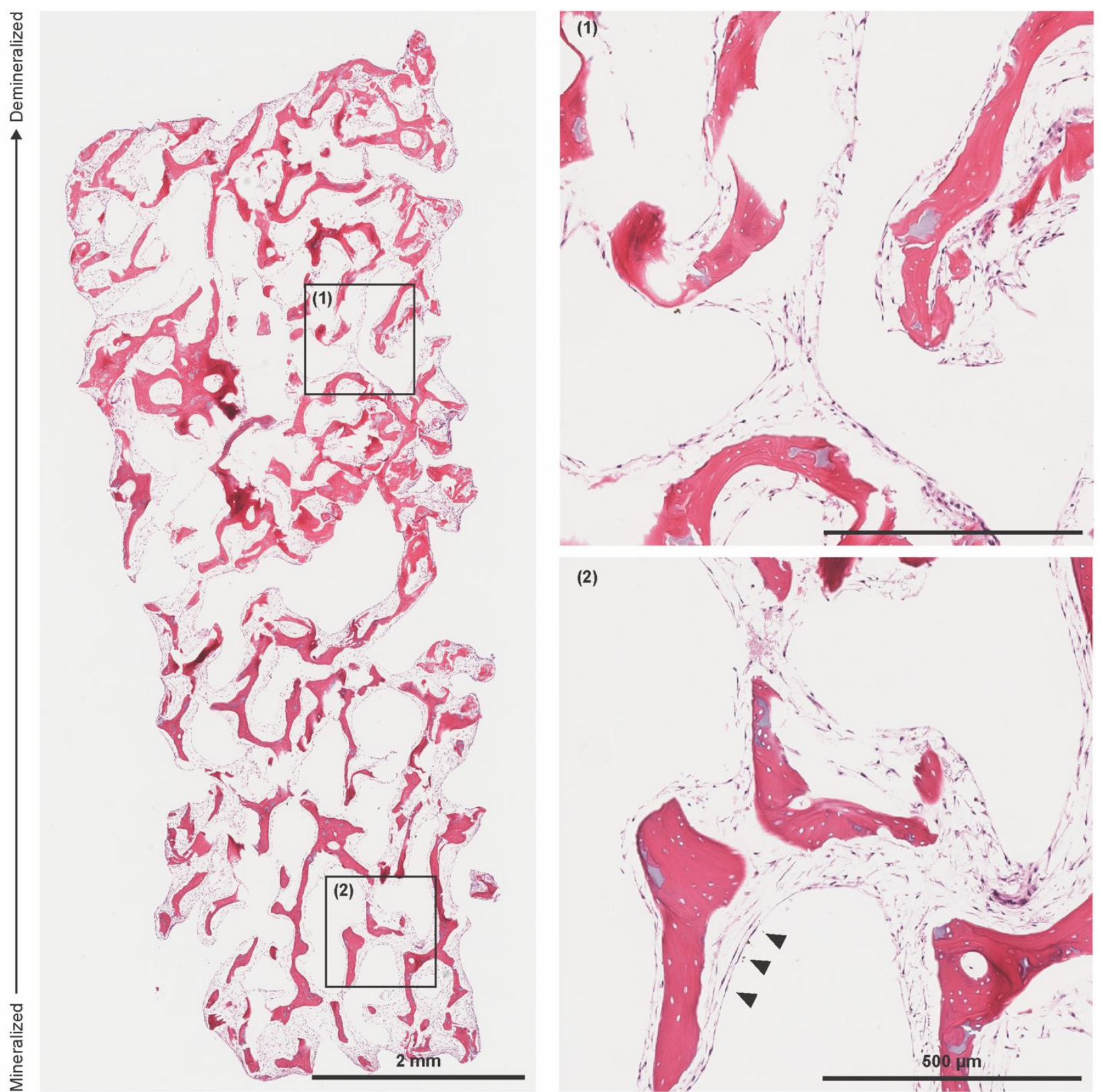

(2)

Figure S10. Hematoxylin and eosin stain of scaffold seeded with MSCs and cultured for 4 days. (1) Demineralized region of scaffold, showing MSCs with rounded phenotypes. (2) Mineralized region of scaffold, showing MSCs elongated along edges of scaffold. Arrows indicate elongated cells in mineralized region of scaffold.

Movie 1. Movie of $\mu \mathrm{CT}$ scan, showing mineralization front using data shown in Figure 2. 